ITP-SB-97-53

hep-th/9709082

\title{
The Implicit Metric on a Deformation of the Atiyah-Hitchin manifold
}

\author{
Gordon Chalmers" \\ Institute for Theoretical Physics \\ State University of New York \\ Stony Brook, NY 11794-3840, USA
}

\begin{abstract}
Using twistor methods we derive a generating function which leads to the hyperkähler metric on a deformation of the Atiyah-Hitchin monopole moduli space. This deformation was first considered by Dancer through the quotient construction and is related to a charge two monopole configuration in a completely broken $S U(3)$ gauge theory. The manifold and metric are the first members of a family of hyperkähler manifolds which are deformations of the $D_{k}$ rational singularities of $C^{2}$.
\end{abstract}

\footnotetext{
${ }^{1}$ E-mail:chalmers@insti.physics.sunysb.edu
} 


\section{Introduction}

The Atiyah-Hitchin metric on the moduli space of two centered monopoles in an $S U(2)$ broken gauge theory [1] has played a crucial role in considerations of S-duality in field and string theory as well as in studies of monopole dynamics [2, 3]. In [4] Dancer considered a family of deformations of the AtiyahHitchin metric. He found these deformations by a hyperkähler quotient from the moduli space of Nahm equations with appropriate bondary conditions. Although the hyperkähler quotient constuction is a very powerful tool for establishing existence and certain properties of hyperkähler manifolds, an explicit solution for the metric in this approach is in general too difficult. In this paper we resort to the second approach to constructing hyperkähler manifolds, the twistor approach, see e.g. [5]. In [6] it has been shown how one may use a twistor description to generate certain classes of metrics through the generalized Legendre transform [7]. The Dancer manifold is in the class of manifolds accessible by generalized Legendre transform and we utilize this technique to compute the metric implicitly.

Our main result is the generating function used to compute the metric in the generalized Legendre transform:

$$
\begin{gathered}
F(z, \bar{z}, v, \bar{v}, w)=-\frac{1}{2 \pi i} \oint_{0} \frac{d \zeta}{\zeta^{3}} \eta(\zeta)+n \oint_{C} \frac{d \zeta}{\zeta^{2}} \sqrt{\eta} \\
+\frac{1}{2 \pi i} \oint_{c_{+}} \frac{d \zeta}{\zeta^{2}}(\sqrt{\eta}+\tilde{b}) \ln (\sqrt{\eta}+\tilde{b})+\frac{1}{2 \pi i} \oint_{c_{-}} \frac{d \zeta}{\zeta^{2}}(\sqrt{\eta}-\tilde{b}) \ln (\sqrt{\eta}-\tilde{b}),
\end{gathered}
$$

where $\eta(\zeta)=z+v \zeta+w \zeta^{2}-\bar{v} \zeta^{3}+\bar{z} \zeta^{4}$; the contour integrals are defined in section 2 . The metrics generated by (四) are also related to the metrics on the moduli space of $(2,1)$ monopoles in a maximally broken $S U(3)$ theory [8].

In section 2, we present the twistor spaces of the families of manifolds which are deformations of the Atiyah-Hitchin manifold. They have a special property which makes them accessible to the generalized Legendre transform construction, which we describe. In particular we have to solve a constraint on the parameters of an elliptic curve lying in the tangent space of $\mathrm{CP}^{1}$. In section 3 we show how the constraint may be solved implicitly by solving for one of the angle coordinates of the parameterization. In section 4 we give a description of further metrics which are deformations of the $D_{k}$ series. We further show how some of these metrics are related to monopole moduli space metrics. 


\section{Twistor Spaces for Dancer's family}

A description of the Atiyah-Hitchin manifold is through the curve in $C^{3}$

$$
x^{2}+y^{2} z=1 .
$$

The metric on which is computed in [1]. Consider next the following family of complex surfaces in $C^{3}$

$$
x^{2} z+(y z+a)^{2}=z+a^{2} .
$$

When $a=0$ we regain the description in (2). In (3) $x, y$ and $z$ are coordinates on $C^{3}$ and $a$ is a free parameter. The whole family in (3) is related to the ones considered by Dancer [4].

In order to put hyperkähler metrics on the set of curves in (3) we start with a family of 3-manifolds $T_{I}$, which will serve as twistor spaces for the the hyperkähler metrics. These twistor spaces are constructed from the holomorphic line bundle $\mathcal{O}(4)$ over $\mathrm{CP}^{1}$ in a manner similar to the construction of the twistor space to the Atiyah-Hitchin metric in [1]. The defining equation for a 3-manifold $T_{I}$ is:

$$
x^{2}(\zeta) \eta(\zeta)+(y(\zeta) \eta(\zeta)+p(\zeta))^{2}=\eta(\zeta)+p^{2}(\zeta) .
$$

In the last equation (4), however, the interpretation of $x, y$, and $\eta$ is different. Namely, $\zeta$ is the standard coordinate on one patch of $\mathrm{CP}_{1}$ and $\eta(\zeta)$ is a section of the holomorphic line bundle $\mathcal{O}(4)$ (i.e. fourth order polynomial in $\zeta$ ). To every section $\eta(\zeta)$ we will frequently associate the following elliptic curve $\mathcal{E}$ in $\mathcal{O}(2): \gamma^{2}=\eta(\zeta)$.

To describe the meaning of the coordinates $x(\zeta)$ and $y(\zeta)$ we need to consider the holomorphic tangent bundle $T \mathrm{CP}_{1}$ of $\mathrm{CP}_{1}$ covered in a standard way by two patches around $\zeta=0$ and $\zeta=\infty$. Let $L^{m}$ be the holomorphic line bundle over $T \mathrm{CP}_{1}$ with transition function $e^{-m \kappa / \zeta}$ with $\kappa$ the coordinate on the fiber. We also define $L^{m}(t)$ to be the line bundle $L^{m} \times \pi^{*} \mathcal{O}(t)$ where the second term in the product is the pullback bundle from $\mathrm{CP}_{1}$. Then the combinations

$$
\eta_{ \pm}(\zeta)= \pm i x(\zeta) \sqrt{\eta(\zeta)}+y(\zeta) \eta(\zeta)+p(\zeta)
$$

are sections of $L^{ \pm m}(2)$. Finally the deformation is given by $p(\zeta) \in \Gamma \mathcal{O}(2)$; its square is on the same footing as $\eta(\zeta)$ and is explicitly parameterized by $p(\zeta)=a+b \zeta-\bar{a} \zeta^{2}$ with $b$ real. 
We recall that the reality involution on the sections of holomorphic line bundles $\mathcal{O}(2 n)$ is

$$
\bar{\eta}^{(2 n)}(\zeta)=(-1)^{n}(\bar{\zeta})^{2 n} \eta^{(2 n)}(-1 / \bar{\zeta}),
$$

and on the twistor space restricts the section $\eta \in \Gamma \mathcal{O}(4)$ to depend on five real parameters (instead of five complex parameters); it also interchanges $p_{+}$and $p_{-}$in (5). Moreover the reality condition enforces the specific form of the section $p(\zeta)$ above with $b \in R, a \in C$. Thus $I=\{a, b\}$ labels the different members of the family of twistor spaces $T_{I}$. Restricting to the fibre over $\zeta=0$ we see that the parameter $a$ labels twistor spaces for different surfaces (3) (labeled also by $a$ ) and for fixed $a$, the parameter $b$ labels different hyperkähler structures on the same surface.

The last ingredient in the description of the twistor spaces $T_{I}$ is the holomorphic 2-form. We will define it to be

$$
\omega=d \eta(\zeta) \wedge d\left(\frac{1}{\sqrt{\eta(\zeta)}} \ln \frac{\eta_{+}(\zeta)}{\eta_{-}(\zeta)}\right)
$$

in accordance with [1] and [1].

As a first step toward recovering the metric we would like to identify a four parameter family of twistor lines. The coefficients of the section $\eta(\zeta) \in \Gamma \mathcal{O}(4)$ provide five parameters and there should be one restriction on them coming form the twistor space equation. Indeed, fix the coefficients of $\eta(\zeta)$ and consider the zeros of the right hand side of $(\mathbb{\Psi})$. These correspond to eight points on the elliptic curve $\mathcal{E}$. According to equation (14) and the meaning of $\eta_{ \pm}$the divisor of four of the points should correspond to a section of the holomorphic line bundle $L^{m}$ restricted to the elliptic curve. The question of how to split the eight zeros in two groups is governed by the real structure. The divisor condition on the second group of zeros is automatically satisfied if the condition on the divisor of the first group is. This gives one condition on the coefficients of $\eta(\zeta)$. If we fix the four remaining parameters in $\eta(\zeta)$ and vary $\zeta$ we can recover the sections $\eta_{ \pm}$and this gives us a twistor line. The solution of the constraint on the parameters of $\eta(\zeta)$ will ocupy the next section and we close this section with some additional remarks.

Rational transformations on the $\mathrm{CP}_{1}$ coordinate

$$
\zeta \mapsto \tilde{\zeta}=\frac{a \zeta+b}{-\bar{b} \zeta+\bar{a}}, \quad|a|^{2}+|b|^{2}=1,
$$


under which all sections transform covariantly are easily seen to be holomorphic maps from the twistor space of a particular surface with given $p(\zeta)$ onto the twistor space of a surface with $\tilde{p}(\tilde{\zeta})=(\bar{b} \tilde{\zeta}+a)^{2} p(\zeta)$. The combination $\tilde{b}^{2}+4 a \bar{a}$ is invariant under these transformations and we conclude that it is enough to consider only the case $a=0, p(\zeta)=\tilde{b} \zeta$ so we have one (real) parameter family of different hyperkähler metrics, whose twistor spaces we will label as $T_{\tilde{b}}$.

There is still $U(1)$ worth of transformations of type (8) namely

$$
\zeta \mapsto \tilde{\zeta}=e^{i \phi} \zeta
$$

which leave every $T_{\tilde{b}}$ invariant. This isometry is not tri-holomorphic and we will illustrate it in section 3 .

\section{The Metric and Constraint Equation}

In this section we will discuss how the deformation of the Atiyah-Hitchin metric may be found through the generalized Legendre transform technique [7]. In order to solve for the metric explicitly we need to specify a constraint on the twistor lines specified by the parameters in $\eta(\zeta)$. In the derivation of the Atiyah-Hitchin metric using the generalized Legendre transform [6] this constraint may be solved for in terms of an overall scale of the coordinates. In the case of the deformation of the Atiyah-Hitchin metric discussed in this work, there is an additional scale coming from the deformation parameter $\tilde{b}$ and we are unable to determine the constraint in this same manner; however, we can determine it in terms of an angular parameter.

Due to the action of the real structure the section $\eta(\zeta)$ has the following form

$$
\eta(\zeta)=z+v \zeta+w \zeta^{2}-\bar{v} \zeta^{3}+\bar{z} \zeta^{4},
$$

with $z \in C, v \in C$, and $w \in R$. The roots of $\eta(\zeta)+p^{2}(\zeta)=0$ then come in complex pairs through the real structure and are denoted $\alpha, \beta,-1 / \bar{\alpha},-1 / \bar{\beta}$. The following four points on the elliptic surface $\mathcal{E}$,

$$
\begin{gathered}
\mathcal{E}:(\alpha, \sqrt{\eta(\alpha)}),(\beta, \sqrt{\eta(\beta)}) \\
(-1 / \bar{\alpha},-\sqrt{\eta(-1 / \bar{\alpha})}),(-1 / \bar{\beta},-\sqrt{\eta(-1 / \bar{\beta})}),
\end{gathered}
$$


then are the zeros of the section $\eta_{+}$. The condition on the divisor then could be written as

$$
\left(\int_{\alpha}^{\infty}+\int_{\beta}^{\infty}-\int_{-1 / \bar{\alpha}}^{\infty}-\int_{-1 / \bar{\beta}}^{\infty}\right) \frac{d \zeta}{\sqrt{\eta(\zeta)}}=2
$$

The integral representation in (12) is defined by an analytic continuation. There is a square root branch cut of the integrand which we take to run between the zeros $\alpha$ and $-1 / \bar{\beta}$ (and $-1 / \bar{\alpha}$ and $\beta$ ). Due to the branch cut the ambiguity is given by integrals along these pairs of zeros; they give complete elliptic integral contributions to (12). This ambiguity will be fixed by comparing the metric to its known asymptotic form.

Alternatively, the constraint may be expressed as the derivative $F_{w}=0$ of the function

$$
\begin{gathered}
F(z, \bar{z}, v, \bar{v}, w)=-\frac{1}{2 \pi i} \oint_{0} \frac{d \zeta}{\zeta^{3}} \eta(\zeta)+n \oint_{C} \frac{d \zeta}{\zeta^{2}} \sqrt{\eta} \\
+\frac{1}{2 \pi i} \oint_{c_{+}} \frac{d \zeta}{\zeta^{2}}(\sqrt{\eta}+\tilde{b}) \ln (\sqrt{\eta}+\tilde{b})+\frac{1}{2 \pi i} \oint_{c_{-}} \frac{d \zeta}{\zeta^{2}}(\sqrt{\eta}-\tilde{b}) \ln (\sqrt{\eta}-\tilde{b})
\end{gathered}
$$

The first term in (13) is given by a contour integral around the origin and the second one $C$ is defined by an integral around one of two branch cuts running between the pairs of zeros $\left(\alpha_{0},-1 / \bar{\beta}_{0}\right)$ and $\left(-1 / \bar{\alpha}_{0}, \beta_{0}\right)$ to the equation $\eta(\zeta)=$ 0 . The remaining two contours $c_{ \pm}$are specified by the zeros of the equation $\eta(\zeta)+\tilde{b}^{2} \zeta^{2}=0$. Explicitly one may write them as line integrals on a sheet of the double cover of the complex plane specified by branch cuts running through the between the zeros of $\eta(\zeta)+\tilde{b}^{2} \zeta^{2}=0$. If $\tilde{b}=0$ then these integrals would be complete elliptic integrals. The representation of the twistor space constraint in (13) defines the generating function used for computing the metric in the Legendre transform technique. By construction it satisfies

$$
\frac{\partial}{\partial w_{a}} \frac{\partial}{\partial w_{b}} F=\frac{\partial}{\partial w_{(a+c)}} \frac{\partial}{\partial w_{(b-c)}} F
$$

where the coordinates $w_{j}$ are defined through $\eta^{(4)}(\zeta)=\sum_{j=0}^{4} w_{j} \zeta^{j}$

In order to solve the constraint (13), we are faced with the problem of adding together two incomplete elliptic integrals of first kind. Unfortunately 
we have not been able to solve the constraint equation explicitly for a scale parameter (for example, the variable $w$ ) as is done in finding the AtiyahHitchin metric in this formulation [6].

We take a rational transformation which sends one of the roots of (10) to infinity and then the transformed quartic is

$$
\tilde{b}^{2}\left[-a \bar{b} \zeta^{2}+(a \bar{a}-b \bar{b}) \zeta+b \bar{a}\right]^{2}=r_{1} \zeta^{3}-r_{2} \zeta^{2}-r_{1} \zeta
$$

Here $r_{1}$ and $r_{2}$ are both real numbers. The zeros of (15) are $x_{1}, x_{2}$ and $-1 / \bar{x}_{1}$, $-1 / \bar{x}_{2}$. The limit $\tilde{b} \rightarrow 0$, which generates the Atiyah-Hitchin curve, is subtle because in this case two of the roots tend to zero and infinity. In (15) the five parameters given by the coefficients of the original section $\eta(\zeta)$ in (10) have been traded in exchange for the three angles describing the $\zeta$ rotation and $r_{1}$ and $r_{2}$.

The constraint on the twistor space is alternatively found by adding together the elliptic integrals in (13) directly. Using the identity (36) after inverting the integrals in (13) we obtain a second form

$$
x-\frac{r_{2}}{3 r_{1}}=\wp\left(m \sqrt{r_{1}} / 2 ; g_{2}, g_{3}\right),
$$

where $x$ is the root of the cubic equation in (15) which goes to infinity when $r_{1} \rightarrow 0$ and $\wp$ is the Weierstrauss function. The modular parameters are

$$
g_{2}=4\left\{1+3\left(\frac{r_{2}}{3 r_{1}}\right)^{2}\right\}
$$

and

$$
g_{3}=4 \frac{r_{2}}{3 r_{1}}\left\{1+2\left(\frac{r_{2}}{3 r_{1}}\right)^{2}\right\} \text {. }
$$

The equation (16), when regarded as giving $r_{1}, r_{2}$, or the ratio $r_{2} / r_{1}$ in (16), is transcendental and may not be solved for explicitly. However, in principle we may solve for one of the angular coordinates appearing in $x$ in (16) through the equation (15); this form is useful for a numerical implementation of describing the metric. In the appendix we express the function in (13) in terms of the rotated coordinates. 


\section{$3.1 \quad U(1)$ Isometries}

In the presence of isometries the construction of the metrics using the generalized Legendre transform may be simplified. In this section we describe how a $U(1)$ isometry, both tri-holomorphic and non-tri-holomorphic, demonstrates this. In the process we will derive a theorem due to Boyer and Finley [10].

In the case of a tri-holomorphic isometry one may always choose a set of complex coordinates so that for a vector field $\psi$ and Kahler form $K$ [5],

$$
\xi=\left(\partial_{u}-\partial_{\bar{u}}\right) \quad \xi K=0 .
$$

Furthermore, if the isometry is only a generic $U(1)$ we may find coordinates $u^{\prime}$ and $\bar{u}^{\prime}$ such that

$$
\psi=\left(u^{\prime} \partial_{u^{\prime}}-\bar{u}^{\prime} \partial_{\bar{u}^{\prime}}\right) \quad \psi K=0
$$

or with a parameterization $u=e^{i \theta} r$

$$
\partial_{\theta} K=0 \text {. }
$$

The former case is special in that metrics possessing a tri-holomorphic $U(1)$ isometry are always constructed locally from an $\mathcal{O}(2)$ coordinate: The Kahler potential always satisfies (19) when written as a function of $u+\bar{u}$. This case, however, is not relevant to our study as both the Atiyah-Hitchin metric and its deformation possess a generic $U(1)$ isometry which is not tri-holomorphic (unless one is in the asymptotic regime). In both cases an $\mathcal{O}(4)$ section is used to formulate the metrics.

First we demonstrate the $U(1)$ action explicitly on the generating function. Recall that the Atiyah-Hitchin metric is found in the generalized Legendre transform from [6]

$$
F_{A H}=-\frac{m}{2 \pi i} \oint_{0} \frac{d \zeta}{\zeta^{2}} \eta(\zeta)+\oint_{C} \frac{d \zeta}{\zeta} \sqrt{\eta(\zeta)},
$$

where the contour $C$ encloses all four roots of $\eta$ as described in [6] (or as given in 13).

Consider a transformation of the coordinate $\eta(\zeta)$ in $(10)$ used to describe these metrics given by a constant phase shift $\zeta \rightarrow e^{i \alpha} \zeta$. Our section transforms as

$$
\eta \rightarrow e^{2 i \alpha}\left(e^{-2 i \alpha} z+e^{-i \alpha} v \zeta+w \zeta^{2}-e^{i \alpha} \bar{v} \zeta^{3}+e^{2 i \alpha} \bar{z} \zeta^{4}\right) .
$$


Upon redefining our coordinates as $z^{\prime}=e^{-2 i \alpha} z$ and $v^{\prime}=e^{-i \alpha} v$ we see explicitly how the generating function remains unchanged for the Atiyah-Hitchin metric. The invariance of the generating function for the Atiyah-Hitchin metric $F_{\mathrm{AH}}$ is due to its particular functional form: The measure of the integrand transforms in the opposite manner and eliminates the phase $e^{i \alpha}$ from appearing explicitly. Of course, the roots to $\eta(\zeta)+b^{2} \zeta^{2}=0$ change.

The example described in (13) also possesses this $U(1)$ invariance provided the deformation parameter also gets rescaled. This is the isometry listed in (9). In general this invariance exists in further examples of metrics provided that the $F$-functions in such cases remains unchanged.

The invariance under these phase shifts explicitly realizes a $U(1)$ isometry: under this rotation we may choose our coefficient $v$ to be real. The Kahler potential is found from the Legendre transform

$$
K(z, \bar{z}, u, \bar{u})=F(z, \bar{z}, v, \bar{v}, w)-u v-\bar{u} \bar{v} \quad u=F_{v}
$$

after taking into account the constraint $F_{w}=0$. The components of the metric are found by appropriate derivatives of the Kahler potential $\left(d s^{2}=\right.$ $\left.\partial_{z} \partial_{\bar{z}} K(z, \bar{z}, u, \bar{u}) d z \otimes d \bar{z}+\ldots\right)$.

This reality property of $u$ after using the phase invariance of $F$ may be used to explicitly simplify the construction of the Kahler potential in (24). We define the coordinates

$$
v=J e^{i \theta} \quad u=r e^{i \theta}
$$

By using the isometry we can reduce the Legendre transform, which usually requires two parameters, to only one

$$
K(z, \bar{z}, r)=F(z, \bar{z}, J)-J r \quad r=2 \partial_{J} F(z, \bar{z}, J) .
$$

The Kahler potential is independent of $\theta$ and we obtain the previous result of Boyer and Finley (20), which is essentially (26).

\section{4 (2,1) Charge Monopole Moduli Space}

Although we are unable to compute the metric explicitly from the generating function, in the following we note its relations to the metric on the $(2,1)$ 
centered moduli space occuring in a completely broken $S U(3)$ gauge theory recently considered in 8 .

The asymptotic form of the metric generated by (13) is found by taking the limit $\eta(\zeta) \rightarrow \tilde{\eta}^{2}(\zeta)$, where $\tilde{\eta}^{2}(\zeta)=z+x \zeta-\bar{z} \zeta^{2}$ (i.e., a coordinate of the $O(2)$ type). This limit has been discussed in both [6, 9] and we do not repeat the analysis here. In the asymptotic regime the metric is (with $m=1$ )

$$
\begin{aligned}
& d s^{2}=\left(1-\frac{n}{|\vec{r}|}+\frac{1}{|\vec{r}-\vec{\lambda}|}+\frac{1}{|\vec{r}+\vec{\lambda}|}\right) d \vec{r} \cdot d \vec{r} \\
& +\left(1-\frac{n}{|\vec{r}|}+\frac{1}{|\vec{r}-\vec{\lambda}|}+\frac{1}{|\vec{r}+\vec{\lambda}|}\right)^{-1} d \tilde{\phi} d \tilde{\phi} .
\end{aligned}
$$

where $\vec{\lambda}=\left(\frac{1}{\sqrt{2}} \operatorname{Re} a, \frac{1}{\sqrt{2}} \operatorname{Im} a, b\right)$. The frame $d \tilde{\phi}$ is given by $d \tilde{\phi}=d \xi+\vec{w} \cdot d \vec{r}$ with

$$
\vec{\nabla} \times \vec{w}=-\vec{\nabla}\left(\frac{n}{|\vec{r}|}-\frac{1}{|\vec{r}-\vec{\lambda}|}-\frac{1}{|\vec{r}+\vec{\lambda}|}\right) .
$$

In the preceeding section we have used an $S O(3)$ rotation to set $a=\bar{a}=0$ : It is shown in [4] that these rotations act on the spaces $M(\vec{\lambda})$ by rotating the perturbation, $M(R \cdot \vec{\lambda})$. The form (27) is the asymptotic form of the (double cover) of the Atiyah-Hitchin metric once we set $\vec{\lambda}=0$. The general perturbation we have in (27) is related to the form of the multi-center TaubNUT class of metrics [11.

In fact, if we replaced the generating function in (13) with a summation over $k$ distinct points $b_{j}$ as in

$$
\begin{gathered}
F^{(k)}(z, \bar{z}, v, \bar{v}, w)=-\frac{1}{2 \pi i} \oint_{0} \frac{d \zeta}{\zeta^{3}} \eta(\zeta)+n \oint \frac{d \zeta}{\zeta^{2}} \sqrt{z}(\zeta) \\
+\sum_{j=1}^{k} \frac{1}{2 \pi i} \oint_{c_{+, j}} \frac{d \zeta}{\zeta^{2}}(\sqrt{z}(\zeta)+b) \ln (\sqrt{z}(\zeta)+b) \\
+\sum_{j=1}^{k} \frac{1}{2 \pi i} \oint_{c_{-, j}} \frac{d \zeta}{\zeta^{2}}(\sqrt{z}(\zeta)-b) \ln (\sqrt{z}(\zeta)-b),
\end{gathered}
$$


then we would obtain the asymptotic form of a $2 k+1$-multi-center Taub-NUT metric and is symmetric under $\vec{r}=-\vec{r}$. The asymptotic form of the monopole moduli space metrics with charges $(2,1, \ldots, 1)$ in the center-of-mass of the $(2$,$) charge has the form (29). The metrics in (29) are the NUT-perturbations$ of the $D_{k}$ space of metrics for $n=0$ (the $D_{k}$ spaces of metrics within the generalized Legendre transform were described in [6] $)$; the first member $k=1$ of which is related to the deformation of the Atiyah-Hitchin metric after a discrete quotient by a $Z_{2}$.

In [8] it was shown using Nahm's equations that the Dancer's family of metrics is the same as that describing the $S U(3) \rightarrow U(1)^{2}$ monopole monopole moduli space with charges $(2,1)$ after taking the infinite mass limit of the $(, 1)$ charge. The three parameters $(a, \bar{a}, b)$ labelling the deformation is now the position of the fixed $(, 1)$ charge. In our derivation we have used an $S O(3)$ rotation to send $a=\bar{a}=0$ and expressed the metric in the center of mass of the $(2$,$) charges. We find that by comparing the asymptotic behavior$ of the "fixed" monopole moduli space metric considered by Houghton that the Dancer's metric is determined by specifying $n=1$ in (13).

Finally, we relate the construction above to generate implicitly the metrics on the moduli spaces of the $(2,1)$ monopoles. The parameters $\vec{\lambda}$ label both the perturbation in the Dancer's metrics or alternatively the positions of the fixed $(, 1)$ charge. As noted in [9] if we allow this position to vary then in principle we may also construct the $(2,1)$ metric by promoting the parameters in $p(\rho)=a+b \rho-\bar{a} \rho^{2}$ to coordinates. In this case the generating functions described above would give the metric on the 8-dimensional space which corresponds to this moduli space metric in the center of mass of the $(2$,$) charge. The moduli space metrics described in 29$ follow from the construnction using Nahm's equations by [8] and prove the form conjectured in [9].

\section{Discussion}

We have described the deformation of the Atiyah-Hitchin metric as well as the NUT-version of the ALE $D_{1}$ space implicitly through the generalized Legendre transform technique. These metrics are of interest for both their relations to problems in monopole dynamics and duality in field theory.

It would be interesting to further explore the detailed form of the defor- 
mation of the Atiyah-Hitchin metric which was implicitly described in this work. It has been used to describe the moduli space of vacua for an $S U(2)$ $N=4$ supersymmetric gauge theory in three dimensions with one hypermultiplet. Such moduli spaces are related by dimensional reduction to $N=2$ gauge theory moduli spaces in four dimensions which possess singularities describing massless mutually non-local dyons.

Last, the Legendre transform technique has been quite useful in describing complete hyperkähler metrics; it is known that all $4 k$-dimensional metrics with $k$ tri-holomorphic isometries may be generated in this formulation. There remains the question on whether the generalized Legendre transform is as extensive in the classification of hyperkähler metrics not possessing the tri-holomorphic isometries. The monopole moduli space metrics do not have these isometries and it would be interesting to find their construction implicitly at the level of the generating function.

Further work in the construction of the moduli space metrics is in progress. One natural outcome is in the construction of all the ALE space metrics as found in the twistor description described in [1]. One example of such a construction is in the higher dimensional analogs of the $A_{k}$ series, which have recently appeared in the form of the $(1,1, \ldots, 1)$ charge metrics in the higher rank gauge groups. However,another example consists in thinking of the $D_{2}$ ALE metric as removing the "NUT" addition to the generating function describing the Atiyah-Hitchin metric. Further examples follow from the higher charge monopole modulit space metrics. In addition, most remaining ALE spaces (and their higher dimensional analogs) have natural embeddings in the work, for example, recently performed on symmetric configurations of monopoles [12].

\section{Acknowledgements}

G.C. thanks I.T. Ivanov for collaboration and Martin Rocek for discussions. This work was supported in part by NSF grant No. PHY 9722101. 


\section{Appendix}

The addition formula for a quartic of general type as in (10) is very cumbersome and we will use a rational transformation (8) on $\zeta$ to put the quartic into a simpler form.

We rotate the quartic (12) into Weierstrauss form by using the $S L(2, C)$ transformation in (8), under which we have

$$
\eta(\zeta) \rightarrow \frac{1}{(-\bar{b} \zeta+\bar{a})^{4}}\left(r_{1} \zeta^{3}-r_{2} \zeta^{2}-r_{1} \zeta\right) \quad d \zeta \rightarrow \frac{d \zeta}{(-\bar{b} \zeta+\bar{a})^{2}}
$$

and

$$
p(\zeta) \rightarrow \frac{\tilde{b}}{(-\bar{b} \zeta+\bar{a})^{2}}(a \zeta+b)(-\bar{b} \zeta+\bar{a})
$$

The quartic $\eta(\zeta)-\tilde{b}^{2} \zeta^{2}=0$ is then placed in the form (15).

Upon shifting the integrand by $\zeta \mapsto \zeta-r_{2} / 3 r_{1}$ we obtain the constraint in the form

$$
z_{1}+z_{2}-\tilde{z}_{1}-\tilde{z}_{2}=\frac{m}{2} \sqrt{r_{1}}
$$

where

$$
z_{1}=\wp^{-1}\left(x_{1}-\frac{r_{2}}{3 r_{1}}\right) \quad \tilde{z}_{1}=\wp^{-1}\left(-\frac{1}{\bar{x}_{1}}-\frac{r_{2}}{3 r_{1}}\right) .
$$

The values for $z_{2}$ are give by replacing $x_{1}$ with $x_{2}$ in (33). In the above we have used the definition of the Weierstrauss $\wp$-function,

$$
z=\int_{\wp(z)}^{\infty} \frac{d \zeta}{\left(4 \zeta^{3}-g_{2} \zeta-g_{3}\right)^{1 / 2}} .
$$

The modular parameters of the Weierstrauss $\wp$-functions in (33) are given in (18).

In the following we shall moderately simplify the form in (32). Due to the reality condition we have the immediate identity

$$
z_{1}+z_{2}=-\tilde{z}_{1}-\tilde{z}_{2}=m \sqrt{r_{1}}
$$


The value of $z_{1}$ may be determined from $\tilde{z}_{1}$ through (33) and (35). The standard addition formula of $\wp$-functions gives

$$
\wp\left(z_{1}+z_{2}\right)=\wp\left(z_{1}\right)+\wp\left(z_{2}\right)+\frac{1}{4}\left[\frac{\wp^{\prime}\left(z_{1}\right)-\wp^{\prime}\left(z_{2}\right)}{\wp\left(z_{1}\right)-\wp\left(z_{2}\right)}\right]^{2} .
$$

Using this identity and the defining differential equation of the $\wp$-function,

$$
\wp^{\prime 2}(z)=4 \wp^{3}(z)-g_{2} \wp(z)-g_{3},
$$

we further obtain the form of the constraint in the form

$$
\wp\left(m \sqrt{r}_{1} ; g_{2}, g_{3}\right)=-\left(x_{1}+x_{2}\right)+\frac{2 r_{2}}{3 r_{1}}+\frac{\tilde{b}^{2}}{r_{1}}\left\{-a \bar{b}\left(x_{1}+x_{2}\right)+a \bar{a}-b \bar{b}\right\}^{2},
$$

in terms of two roots to (15) which are not conjugates to one another (i.e. $\left.x_{1} \neq-1 / \bar{x}_{2}\right)$.

We end the appendix with some formulas expressing the generating function in terms of the coordinates $r_{1}, r_{2}$ and $a, b$. The first term in (13) is

$$
\begin{gathered}
F_{1}=\frac{1}{2 \pi i} \oint \frac{d \zeta}{\zeta^{2}} \sqrt{\eta}(\zeta) \ln (\sqrt{\eta} \pm b) \\
=\frac{\sqrt{r}_{1}}{2(a \bar{b})^{2}} \int_{z_{1}}^{-1 / \bar{z}_{1}} d z \frac{\wp^{\prime}(z)^{2}}{\left[\left(\wp(z)+\frac{r_{2}}{3 r_{1}}+\frac{b}{a}\right)\left(\wp(z)+\frac{r_{2}}{3 r_{1}}-\frac{\bar{a}}{b}\right)\right]^{2}},
\end{gathered}
$$

evaluated between the zeros of $\sqrt{z} \pm b=0$. The form (39) rewritten into,

$$
F_{1}=\frac{\sqrt{r_{1}}}{2} \int_{z_{1}}^{-1 / \bar{z}_{1}} d z\left\{\frac{d}{d z} \ln \left(\frac{\wp(z)+\frac{r_{2}}{3 r_{1}}+\frac{b}{a}}{\wp(z)+\frac{r_{2}}{3 r_{1}}-\frac{\bar{a}}{b}}\right)\right\}^{2} .
$$

The second contribution is a total derivative,

$$
F_{2}=\frac{1}{2 \pi i} \oint \frac{d \zeta}{\zeta^{2}} b \ln (\sqrt{\eta} \pm b)
$$

which after the $S L(2, C)$ rotation is

$$
F_{2}=-\frac{\tilde{b}}{a \bar{b}} \int_{z_{1}}^{-1 / \bar{z}_{1}} d z \frac{\wp^{\prime}(z)}{\left[\left(\wp(z)+\frac{r_{2}}{3 r_{1}}+\frac{b}{a}\right)\left(\wp(z)+\frac{r_{2}}{3 r_{1}}-\frac{\bar{a}}{b}\right)\right]} .
$$


The integral (41) may be expressed as a total derivative. The result is

$$
F_{2}=-\left.\tilde{b} \ln \left[\frac{\wp(z)+\frac{r_{2}}{3 r_{1}}+\frac{b}{a}}{\wp(z)+\frac{r_{2}}{3 r_{1}}-\bar{a}}\right]\right|_{z_{1}} ^{-1 / \bar{z}_{1}},
$$

evaluated at the end-points of the integral in (41). These evaluations of the generating function will be useful when constructing the metric using the formulas [7] and a possible numerical evaluation.

\section{References}

[1] M. Atiyah and N. Hitchin, The geometry and dynamics of magnetic monopoles., Princeton, NJ (1988).

[2] A. Sen, Phys. Lett. B329:217 (1994), hep-th/9402032.

[3] N. Seiberg, E. Witten, hep-th/9607163.

[4] A.S. Dancer, Quart. J. Math. Oxford 45:463 (1994).

[5] N. J. Hitchin, A. Karlhede, U. Lindstrom, M. Roček, Comm. Math. Phys. 108:535 (1987).

[6] I.T. Ivanov and M. Roček, Comm. Math. Phys. 182:291 (1996), hepth/9512075.

[7] M. Roček, U. Lindstrom, Comm. Math. Phys. 115:21 (1988).

[8] C.J. Houghton, hep-th/9702161.

[9] G. Chalmers, Phys. Rev. D, hep-th/9605182.

[10] C.P. Boyer, F.D. Finley, J. Math. Phys. 23:1126.

[11] S.W. Hawking, Phys. Lett. 60A:8 (1977).

[12] N.S. Manton, M.K. Murray, hep-th/9407102 . 